\title{
Commentary: The power (under control) of meta-analysis in the synthesis of clinical knowledge
}

\author{
Luca Bertolaccini, MD, PhD, FCCP
}

\author{
From the Department of Thoracic Surgery, Maggiore Teaching Hospital, Bologna, Italy. \\ Disclosures: Author has nothing to disclose with regard to commercial support. \\ Received for publication Jan 13, 2019; accepted for publication Jan 14, 2019; available ahead of print Feb 20, \\ 2019. \\ Address for reprints: Luca Bertolaccini, MD, PhD, FCCP, Department of Thoracic Surgery, Maggiore Teaching \\ Hospital, Largo Nigrisoli 2, 40133 Bologna, Italy (E-mail: luca.bertolaccini@gmail.com). \\ J Thorac Cardiovasc Surg 2019;158:1101-2 \\ $0022-5223 / \$ 36.00$ \\ Copyright (c) 2019 by The American Association for Thoracic Surgery \\ https://doi.org/10.1016/j.jtcvs.2019.01.062
}

Even if mechanical ventilation reduces the incidence of respiratory failure and improves survival after cardiac surgery, it may lead to ventilator-associated pneumonia. ${ }^{1}$ Shreds of evidence in the medical literature suggest that chlorhexidine gluconate (a broad-spectrum antibacterial agent) could be used to decrease the incidence of ventilator-associated pneumonia after cardiac surgery. ${ }^{2}$ The technique of metaanalysis is designed to increase the sample size and the power of the effects under investigation by combining the results of studies analyzed. ${ }^{3}$ Like many other statistical techniques, meta-analyses are potent tools but must be used carefully, because most data are derived from observational studies and single-center prospective trials, and few data are derived from large-scale multicenter trials. ${ }^{4}$

In their systematic review and meta-analysis published this month in the Journal, Bardia and colleagues ${ }^{5}$ have assessed the efficacy of preoperative chlorhexidine gluconate in the reduction of postoperative pneumonia after cardiac surgery and found that the preoperative mouthwash significantly reduces this risk. From a pathophysiologic point of view, oral decontamination by chlorhexidine could decrease the microbial burden of the aerodigestive tract and thus apply a protective effect, which would be particularly pronounced in the prevention of gram-positive pneumonia. ${ }^{5}$

As recognized by Bardia and colleagues, ${ }^{5}$ this systematic review and meta-analysis evaluated data derived only from cardiac surgeries. The generalizability to noncardiac surgical patients is thus still unclear, with contradictory results presented in the literature for thoracic surgery. For instance, a systematic review recommended the perioperative oral decontamination in adult patients undergoing elective thoracic noncardiac surgery. ${ }^{6}$ In contrast, a recent multicenter randomized controlled double-blind trial showed that chlorhexidine decontamination did not decrease the incidence of ventilator-associated pneumonia. ${ }^{7}$

Unfortunately, the systematic review and meta-analysis of Bardia and colleagues ${ }^{5}$ was not registered in the international database of prospectively recorded systematic

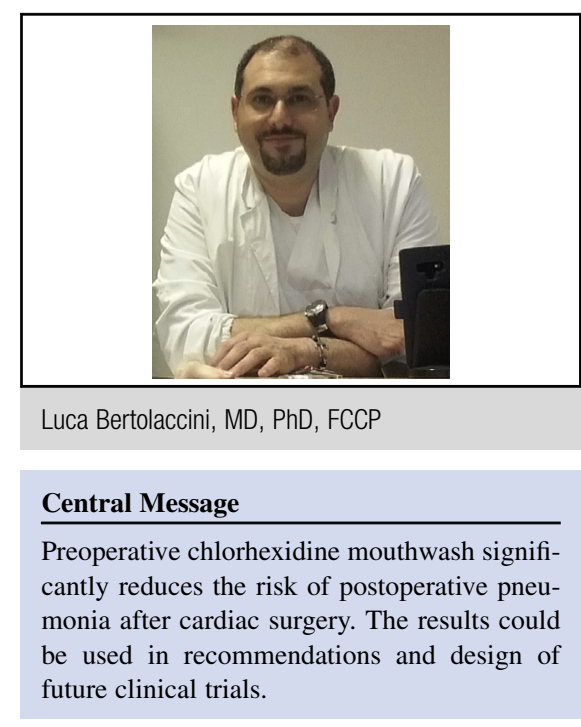

See Article page 1094.

reviews, PROSPERO (www.crd.york.ac.uk/prospero/). This registry provides a comprehensive listing of systematic reviews, with the aim of avoiding replication and decreasing the opportunity for reporting bias. The registration of the systematic review protocol may alert guideline groups that a detailed analysis is being performed and may offer occasions for cooperation with colleagues for implementation of the findings of the investigation. ${ }^{8}$ The registration of the protocol could appear as an additional duty to systematic review and meta-analysis, but it is easy, it reduces repetition of studies, and the review team could benefit from registration by avoiding subsequent uncertainty. ${ }^{9}$ Nonetheless, conducting meta-analyses does not overcome inherent problems in the design and execution of the primary studies, because meta-analyses do not correct publication biases. Therefore, studies reporting remarkable results are more likely to be identified, summarized, and subsequently pooled than studies with smaller effect sizes. ${ }^{10}$

In conclusion, every meta-analysis presents some limitations in the planning, in the execution, and the analysis, due to the heterogeneity in patient populations or the surgical techniques, the different duration of follow-up, and the various definitions of clinical outcomes. Despite these well-known caveats, the results derived from the systematic review and meta-analysis of Bardia and colleagues ${ }^{5}$ could 
be used to provide guidance in the design of future clinical trials and the completion of recommendations about the preoperative use of chlorhexidine for the reduction of postoperative pneumonia after cardiac surgery.

\section{References}

1. Lin YJ, Xu L, Huang XZ, Jiang F, Li SL, Lin F, et al. Reduced occurrence of ventilator-associated pneumonia after cardiac surgery using preoperative $0.2 \%$ chlorhexidine oral rinse: results from a single-centre single-blinded randomized trial. J Hosp Infect. 2015;91:362-6.

2. Nicolosi LN, del Carmen Rubio M, Martinez CD, González NN, Cruz ME. Effect of oral hygiene and $0.12 \%$ chlorhexidine gluconate oral rinse in preventing ventilator-associated pneumonia after cardiovascular surgery. Respir Care. 2014;59:504-9.

3. Walker E, Hernandez AV, Kattan MW. Meta-analysis: its strengths and limitations. Cleveland Clin J Med. 2008;75:431-9.
4. Yanagawa B, Tam DY, Mazine A, Tricco AC. Systematic review and metaanalysis in cardiac surgery: a primer. Curr Opin Cardiol. 2018;33:184-9.

5. Bardia A, Blitz D, Dai F, Hersey D, Jinadasa S, Tickoo M, et al. Preoperative chlorhexidine mouthwash to reduce pneumonia after cardiac surgery: a systematic review and meta-analysis. J Thorac Cardiovasc Surg. 2019;158: 1094-100.

6. Pedersen PU, Larsen P, Håkonsen SJ. The effectiveness of systematic perioperative oral hygiene in reduction of postoperative respiratory tract infections after elective thoracic surgery in adults: a systematic review. JBI Database System Rev Implement Rep. 2016;14:140-73.

7. D'Journo XB, Falcoz PE, Alifano M, Le Rochais JP, D'Annoville T, Massard G, et al. Oropharyngeal and nasopharyngeal decontamination with chlorhexidine gluconate in lung cancer surgery: a randomized clinical trial. Intensive Care Med. 2018;44:578-87.

8. PLoS Medicine Editors. Best practice in systematic reviews: the importance of protocols and registration. PLoS Med. 2011;8:e1001009.

9. Chang SM, Slutsky J. Debunking myths of protocol registration. Syst Rev. 2012; $1: 4$.

10. Garg AX, Hackam D, Tonelli M. Systematic review and meta-analysis: when one study is just not enough. Clin J Am Soc Nephrol. 2008;3:253-60. 